\title{
Who should be prioritized for COVID-19 vaccination in China? A descriptive study
}

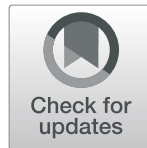

Juan Yang ${ }^{1}$, Wen Zheng ${ }^{1}$, Huilin Shi ${ }^{1}$, Xuemei Yan ${ }^{1}$, Kaige Dong ${ }^{1}$, Qian You', Guangjie Zhong ${ }^{1}$, Hui Gong ${ }^{1}$ Zhiyuan Chen ${ }^{1}$, Mark Jit ${ }^{2,3,4}$, Cecile Viboud ${ }^{5}$, Marco Ajelli, ${ }^{6,7}$ and Hongjie $\mathrm{Yu}^{1,8,9^{*}}$

\begin{abstract}
Background: All countries are facing decisions about which population groups to prioritize for access to COVID-19 vaccination after the first vaccine products have been licensed, at which time supply shortages are inevitable. Our objective is to define the key target populations, their size, and priority for a COVID-19 vaccination program in the context of China.
\end{abstract}

Methods: On the basis of utilitarian and egalitarian principles, we define and estimate the size of tiered target population groups for a phased introduction of COVID-19 vaccination, considering evolving goals as vaccine supplies increase, detailed information on the risk of illness and transmission, and past experience with vaccination during the 2009 influenza pandemic. Using publicly available data, we estimated the size of target population groups, and the number of days needed to vaccinate $70 \%$ of the target population. Sensitivity analyses considered higher vaccine coverages and scaled up vaccine delivery relative to the 2009 pandemic.

Results: Essential workers, including staff in the healthcare, law enforcement, security, nursing homes, social welfare institutes, community services, energy, food and transportation sectors, and overseas workers/students (49.7 million) could be prioritized for vaccination to maintain essential services in the early phase of a vaccination program. Subsequently, older adults, individuals with underlying health conditions and pregnant women (563.6 million) could be targeted for vaccination to reduce the number of individuals with severe COVID-19 outcomes, including hospitalizations, critical care admissions, and deaths. In later stages, the vaccination program could be further extended to target adults without underlying health conditions and children (784.8 million), in order to reduce symptomatic infections and/or to stop virus transmission. Given 10 million doses administered per day, and a twodose vaccination schedule, it would take 1 week to vaccinate essential workers but likely up to 7 months to vaccinate $70 \%$ of the overall population.

Conclusions: The proposed framework is general but could assist Chinese policy-makers in the design of a vaccination program. Additionally, this exercise could be generalized to inform other national and regional strategies for use of COVID-19 vaccines, especially in low- and middle-income countries.

Keywords: Novel coronavirus disease 2019, Vaccination, Target population, China

\footnotetext{
* Correspondence: yhj@fudan.edu.cn

This study does not necessarily represent the views of the US government or the National Institutes of Health.

'School of Public Health, Fudan University, Key Laboratory of Public Health

Safety, Ministry of Education, Shanghai, China

${ }^{8}$ Shanghai Institute of Infectious Disease and Biosecurity, Fudan University,

Shanghai, China

Full list of author information is available at the end of the article
}

C C The Author(s). 2021 Open Access This article is licensed under a Creative Commons Attribution 4.0 International License, which permits use, sharing, adaptation, distribution and reproduction in any medium or format, as long as you give appropriate credit to the original author(s) and the source, provide a link to the Creative Commons licence, and indicate if changes were made. The images or other third party material in this article are included in the article's Creative Commons licence, unless indicated otherwise in a credit line to the material. If material is not included in the article's Creative Commons licence and your intended use is not permitted by statutory regulation or exceeds the permitted use, you will need to obtain permission directly from the copyright holder. To view a copy of this licence, visit http://creativecommons.org/licenses/by/4.0/. The Creative Commons Public Domain Dedication waiver (http://creativecommons.org/publicdomain/zero/1.0/) applies to the data made available in this article, unless otherwise stated in a credit line to the data. 


\section{Background}

The pandemic is causing unprecedented impact on global health and the economy. In the absence of safe and highly effective vaccines and treatment options, nonpharmaceutical interventions are used to decrease transmission and reduce the burden of coronavirus disease 2019 (COVID-19) but most of these interventions have large economic costs [1]. Effective vaccines against COVID-19 are urgently needed to reduce the significant burden of COVID-19 morbidity and mortality. Globally, there are over 274 vaccine candidates at various stages of development in the research pipeline. Of these, 59 candidates have entered clinical trials [2].

On June 26, 2020, the World Health Organization (WHO) unveiled a plan to deliver 2 billion doses of COVID-19 vaccines, of which 50\% will go to low- and middle-income countries, by the end of 2021 [3]. Currently, the projected global production capacity is inadequate to provide COVID-19 vaccines for every human being on the planet, particularly immediately after the first vaccine has been licensed. It is possible that countries and entire regions will have no access to vaccines. For example, COVID-19 cases are rapidly increasing in most African countries [4]. However, none of the COVID-19 vaccine candidates is being developed by an African manufacturer. Even if a vaccine were available, many low-income countries would have to rely on vaccines manufactured abroad. Hence national and multinational vaccine producers will need to allocate a proportion of their production to countries that do not have the financial ability to pre-order vaccine doses that are still to be licensed. Setting priorities for target populations to be vaccinated and optimizing resources within and between countries entails difficult choices. Nonetheless, this is critical for a successful global pandemic vaccination program, and this needs to be addressed urgently. The WHO Strategic Advisory Group of Experts on Immunization (SAGE) Values Framework for The Allocation and Prioritization of COVID-19 Vaccination offers core principles for vaccine distribution [5]. These guidelines need to be further specified and tailored to each county, taking into local contexts including but not limited to the intensity of epidemic, the objectives of pandemic responses, the vaccine supply, and the size of the population eligible for vaccination.

China was the first country to face the COVID-19 pandemic, although only Wuhan, in Hubei Province, was hit by a major wave of infections [6]. Nearly the entire population of mainland China ( 1.4 billion people) is still susceptible to COVID-19. Recent surges of COVID-19 cases occurred in a growing number of cities such as Beijing, Dalian, Urumchi, and Kashgar, following one or more months without any report of locally acquired infections [7]. There is a risk of a new major wave of COVID-19, especially after the economy and society have re-opened both domestically and abroad.

China has invested substantial resources in vaccines and is one of the main actors in the race to develop a vaccine to help control the COVID-19 pandemic, with resources provided by government, manufacturers, and non-governmental organizations [8]. Eighteen vaccine candidates are being developed in mainland China; five of them are in phase III trials as of November 12, 2020 [9]. New COVID-19 vaccine production facilities recently completed or currently under construction are expected to have the capacity to produce 0.61 billion doses by the end of 2020 and further expanded in 2021 [10]. However, the output is far behind the quantity needed to vaccinate a population of nearly 1.4 billion people in mainland China alone (given a two-dose schedule for all vaccine candidates).

The Joint Prevention and Control Mechanism of the State Council roughly divides the target population for COVID-19 vaccination into three groups, including those with high risks of exposures to the novel severe acute respiratory syndrome coronavirus 2 (SARS-CoV-2), those with high-risks of severe outcomes, and the general population, with priority given to the former two groups [10]. In July 2020, three COVID-19 vaccines were licensed in China for emergency use among individuals at high risk of exposure to SARS-CoV-2, including frontline medical personnel and overseas workers in China. Media reports show that over one million people have been vaccinated as of December 1, 2020 [11, 12]. According to recent surveys [13-15], the (general) Chinese population has a high level of willingness to accept COVID-19 vaccination. Hence, with more vaccines expected to be licensed by the end of 2020/early 2021, there is a need to define the priority target groups for a wide-scale COVID-19 vaccination program. This study aims to define the priority target populations, their size, and priority for a phased introduction of COVID-19 vaccination with evolving goals in mainland China, accounting for risk of severe illness and transmission. This approach is generalizable to inform national and regional strategies for the use of COVID-19 vaccines, especially in low- and middle-income countries.

\section{Methods}

\section{Goals of the COVID-19 vaccination program}

Using the Pandemic Severity Assessment Framework [16], developed by the United States (US) Centers for Disease Control and Prevention to determine pandemic influenza severity, the COVID-19 pandemic can be characterized as having both very high transmissibility and clinical severity [17]. The overarching goal of a vaccination program in the midst of such a pandemic is to vaccinate all persons willing to be vaccinated. However, due to limited supplies, prioritization is warranted. The specific goal of COVID-19 
vaccination in China could be determined in a phased approach. In this early phase, the most important objective (primary goal) of the vaccination program is to maintain essential services (e.g., healthcare and national security) $[18,19]$. The second objective (secondary goal) is to reduce the number of individuals with severe outcomes, including hospitalizations, critical care admissions, and deaths $[18,19]$. In later stages, the objective of the vaccination program can be further extended to reduce symptomatic infections and/or to stop virus transmission (tertiary goal). These goals should be adapted along with the evolving dynamic of the epidemic and an increase of vaccine supplies. We accordingly refer to this approach as a phased universal vaccination program.

\section{Priority population groups for a COVID-19 vaccination program}

In line with the aforementioned goals of a COVID-19 vaccination program, prioritization is based on utilitarian (i.e., maximizing health and economic benefit) and egalitarian (i.e., protecting the worst off) principles. We define population groups to be prioritized by occupation, age, and underlying conditions, taking account (1) the interim framework for COVID-19 vaccine allocation and available guidance on allocating vaccines during an influenza pandemic [e.g. from the WHO, US and the United Kingdom (UK), see summary in Additional file 1: Table S1 [5, 18, 20]], (2) the objectives of and experience gained from the $2009 \mathrm{H} 1 \mathrm{~N} 1$ pandemic vaccination program in China [21], (3) specific high-risk groups for severe COVID-19 outcomes and high-risk groups for exposures, and (4) lessons learned from the response to the COVID-19 outbreak in Wuhan such as the role of critical workers in sustaining essential societal functions [1] (Fig. 1). Priority groups include (1) essential workers, including but not limited to healthcare workers (utilitarian principles); (2) high-risk individuals such as those at the highest risk of severe/fatal outcomes (egalitarian principles); (3) individuals who play a key role in transmission (both utilitarian and egalitarian principles) [22].

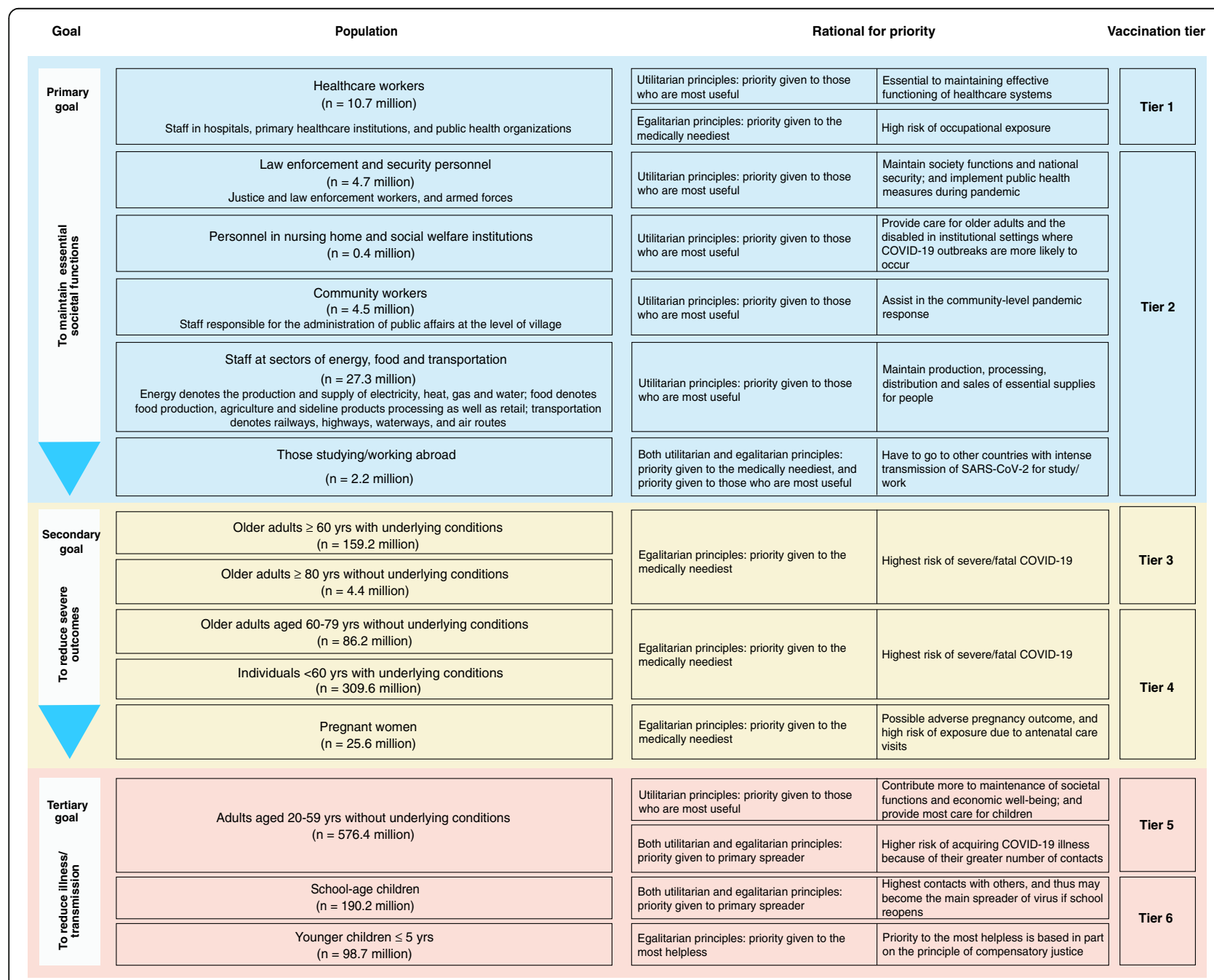

Fig. 1 Prioritized segments of the population for a COVID-19 vaccination program as well as estimated population size 
Generally, within the populations of interest for the primary and tertiary vaccination goals, the target population groups that met $\geq 2$ of the aforementioned principles were assigned to a higher tier. For the secondary goal, the target population at higher risk of severe/fatal COVID-19 outcome was assigned to a higher tier. Subsequently, these population groups were categorized into six vaccination tiers in order of decreasing priority. Across priority population groups, vaccines can be allocated and administered according to tier, which means that all groups within a tier have equal priority for vaccination.

\section{Essential workers}

Individuals who are critical for preserving essential societal functions for public health and safety as well as the well-being of the community during a pandemic include (1) first responders who may have close contact with potential COVID-19 patients in professional settings, including healthcare, public health, and community workers (these include staff in community service agencies, who maintain supply of daily essential needs for people under lockdown, and take routine prevention measures such as fever screening and environmental disinfection); (2) individuals who are essential for maintaining national security, namely individuals working in law enforcement agencies and security personnel (police and military); (3) workers maintaining production and supply of daily essentials, including energy, water, food, and transportation.

Healthcare workers are essential in maintaining an effective healthcare system, not only for COVID-19 but also for other healthcare needs. They often have a high risk of infection due to occupational exposure. For instance, healthcare workers performing endotracheal intubation had a higher risk of SARS-CoV-2 infection than other healthcare workers (odds ratio 4.33, 95\% confidence interval 1.16-16.07) since they had higher exposure associated with aerosol-generating procedures [23]. Public health staff also play a crucial role in the COVID19 response, with responsibilities including, but not limited to, case detection, isolation, tracing, and testing of close contacts, surveillance, and health communication.

An additional category of essential workers includes staff in nursing homes and social welfare institutions, who provide care for older adults and disabled persons in institutional settings, where COVID-19 outbreaks could be devastating [24]. Police are necessary for the society to function and in China are also responsible for implementing a set of public health measures such as tracing of cases and close contacts, and isolation. In addition to maintaining national security, the military also plays a key role in COVID-19 response, as they can provide medical care and support the implementation of prevention and control strategies. Further, community network members are needed to assist in local pandemic response such as mass screening and provide support for vulnerable populations such as seniors, those living alone, and households complying with voluntary quarantine when a household member is ill. The energy (electricity, oil, fuel, and natural gas), water, food, and transportation sectors maintain production, processing, distribution, and sale of essential supplies for the population. These personnel are critical to providing essential goods and services, and thus need to work even during periods of community restrictions, social distancing, or closure orders. Moreover, given the current epidemiological situation in China, characterized by a nearly entirely susceptible population and very limited local transmission, individuals working or studying abroad may have a higher risk of exposure to SARS-CoV-2 compared to domestic residents. As such, this class of individuals has been included in the vaccine emergency programs already implemented in Beijing, Sichuan, and Wuhan and is thus included in Tier 2 in the present study.

Accordingly, we recommend these individuals to be an appropriate first-level priority target group for vaccination. We obtained the population size stratified by occupation from publicly available data, including the China Economic Census Yearbook 2018, the Tabulation the 2010 Population Census of the People's Republic of China, White Paper on China's National Defense, Ministry of Education, Ministry of Commerce, and published literature [25-30].

\section{High-risk individuals}

To meet the secondary goal of the vaccination program, individuals who are at increased risk for severe outcome of COVID-19 could be considered a priority target population for vaccination. We conducted a narrative literature review in PubMed, Embase, Web of Science, medRxiv, and bioRxiv for systematic reviews written in English, to identify the risk factors of severe illness associated with COVID-19. We searched for ("severe" OR "severity" OR "critical" OR "hospitaliz" OR "ICU" OR "death" OR "mortality" OR "fatal") AND ("risk factor") AND ("2019-nCov" OR "COVID-19" OR "COVID 19" OR "2019 novel coronavirus" OR "coronavirus disease 2019" OR "SARS-CoV-2" OR "SARS CoV 2" OR "severe acute respiratory syndrome coronavirus 2 ").

Clark and colleagues extracted the prevalence of underlying health conditions from the Global Burden of Diseases, Risk Factors, and Injuries Study (GBD), and estimated the number of people with at least one of these conditions in 2019 for 188 countries [31]. Using Clark's method, we updated the probability of having at least one of these conditions for China to additionally include the prevalence of body mass index $\geq 30$, which were 
identified as risk factors by our review. Then we estimated the age-specific population size of individuals with any of these conditions by multiplying the estimated probability by the United Nations (UN) mid-year population estimates for 2020 for China [32]. The population size of individuals without these conditions was calculated subtracting those with health conditions from the total population.

Pregnant women were additionally included in the list of high-risk groups. We estimated the number of women who are pregnant in 1 year as the sum of all live births, still births, fetal deaths, and abortions in that year. The number of live births was obtained from China Health Statistical Yearbook in 2020 [33]. The number of still births and fetal deaths was estimated as the product of the number of perinatal deaths and the fraction of those deaths which are still births and fetus deaths (68.59\%) [34]. We estimated the number of abortions by dividing the number of induced abortions by the proportion of induced abortions (88.54\%) [35].

\section{Individuals at high risks of symptomatic COVID-19 infections}

A second narrative literature review was conducted to assess the risk of symptomatic COVID-19 infection, using the search query, ("2019-nCov" OR "COVID-19" OR "COVID 19" OR "2019 novel coronavirus" OR "coronavirus disease 2019" OR "SARS-CoV-2" OR "SARS CoV 2" OR "severe acute respiratory syndrome coronavirus 2") AND ("incidence" OR "attack rate" OR "morbidity") AND ("age profile" OR "age group" OR "age range" OR "age structure" OR "age composition" OR "age spectrum"). Based on the identified risk factors for symptomatic COVID-19 infections, we defined the target populations for vaccination that would help meet the tertiary goal of reducing illness. The populations size was obtained from UN mid-year population estimates for 2020 for China [32], and Ministry of Education of China [36].

\section{Estimating size of target population of the phased universal vaccination program}

First, we estimated the corresponding population size separately for each target population as mentioned above. When a person is included in more than one group, she/ he is intended to be vaccinated in the highest tier group in which she/he is included. Accordingly, we then excluded people in more than one risk group to estimate the total population size stratified by goals of vaccination in different phases of the pandemic, and by vaccination tiers.

Assuming vaccine efficacy (VE) around $85-90 \%$, as preliminary analyses of phase 3 clinical trials of several COVID-19 vaccines seem to suggest [37], and considering the basic reproduction number $\left(R_{0}\right)=2.5$, as found in previous studies about China [38], we used the wellknown eq. $\left(1-1 / R_{0}\right) / \mathrm{VE}$ to estimate the minimum fraction of population to be immunized to reach herd immunity [39]. The resulting estimate of the vaccination coverage is around $70 \%$, in agreement with previous studies [40]. As a sensitive analysis, we considered more conservative estimates of vaccine efficacy (around 65$70 \%$ ) and thus a vaccination coverage of $90 \%$. Those two scenarios align well with estimates of willingness to be vaccinated against COVID-19 in the Chinese population, namely $72.5-91.3 \%$ according to three surveys [13-15]. We therefore estimated the days needed to vaccinate $70 \%$ of the targeted population in the sequence of tiers given a two-dose vaccination schedule, without accounting for issues in production capacity (see schematic diagram in Additional file 1: Fig. S1).

During the 2009 influenza pandemic, a maximum of 3 million daily doses of pandemic influenza H1N1 (H1N1pdm) vaccines were administered in China [41]. However, the willingness to be vaccinated against COVID-19 is higher than that for the 2009 H1N1 pandemic $[13,42]$. Moreover, the vaccine distribution capacity is likely to be improved as well, spurred by the progressive enhancement of the roll-out of Supplementary Immunization Activities in Children in the last decade [43]. As such, we assumed that the capacity of COVID-19 vaccination services could be scaled up to 10 million doses administered per day in the baseline analysis. Sensitivity analyses on the daily doses administered ( 3 and 20 million) were conducted as well. We also conducted a sensitivity analysis using an uptake rate of $90 \%$.

\section{Results}

Figure 1 illustrates the priority population groups and the corresponding population size estimated without excluding duplicates between groups.

\section{Essential workers}

It is important to stress that the vaccine may be in extremely short supply when first available. To meet the primary goal of vaccination, thus it could be necessary to consider healthcare workers as the top priority (Tier 1 of the vaccination strategy) based on utilitarian and egalitarian principles. Law enforcement and security workers, personnel in nursing home and social welfare institutes, community workers, workers in energy, food, and transportation sectors are included in Tier 2 based on utilitarian principles. Those studying/working abroad are also included in Tier 2 based on utilitarian and egalitarian principles (Fig. 1). We estimated that in mainland China there are 10.7 million healthcare workers, 4.7 million people working in law enforcement agencies and security personnel, 0.4 million personnel in nursing home and social welfare institutes, 4.5 million community 
workers, 27.3 million workers in the energy, food, and transportation sectors, and 2.2 million persons studying/ working abroad.

\section{High-risk individuals}

Over 50 published systematic reviews reported the pooled risk of severe outcome of COVID-19 (Additional file 1: Table S2). These reviews showed that an increased risk of severe outcomes from SARS-CoV-2 infection was observed in individuals with chronic respiratory disease, heart disease, cardio-cerebrovascular disease, hypertension, diabetes, chronic renal diseases, chronic liver disease, cancer, and obesity [44-51] (Additional file 1: Table S2). One systematic review evaluated the disease severity of COVID-19 during pregnancy and found that $21 \%$ were severe/critical cases [52]. COVID-19 may cause fetal distress, miscarriage, respiratory distress, and preterm delivery, although evidence for these associations is still inconclusive [53]. Moreover, pregnant women have high frequency of antenatal care visits and thus have a possibly higher exposure to SARS-CoV-2. Although no systematic review found a significantly higher risk of severe outcomes for those with immunodeficiency/immunosuppression, chronic neurological disorders, and sickle cell disorders, we included these categories in our analysis as recommended by the US and UK [47, 54-56].

Age is one of the most important risk factors for severe/ fatal COVID-19. Our systematic reviews showed that individuals age $\geq 60$ years had about 4-fold higher risk of severe/ fatal COVID-19 than younger people (Additional file 1: Table S2). Wu et al. found that the case-fatality risk for those aged $\geq 80$ years was $1.7-3.6$ times that among those aged 70-79 and 60-69 years [57]. Age and underlying conditions combine to increase the risk [58]. Accordingly, adults $\geq 60$ years of age with underlying conditions, and adults $\geq 80$ years of age without underlying conditions, who are at the highest risk of severe/fatal COVID-19, were considered in Tier 3, based on egalitarian principles. Compared to these persons, the risk of severe/fatal COVID-19 among older adults aged 60-79 years without underlying conditions and individuals < 60 years of age with underlying conditions was lower. These individuals aged $<60$ years with pre-existing medical conditions and pregnant women were included in Tier 4 based on egalitarian principles (Fig. 1).

We estimated that 309.6 million individuals aged $<60$ years and 159.2 million individuals aged $\geq 60$ years had at least one high-risk medical condition in mainland China. The number of pregnant women was thus estimated at 25.6 million in mainland China (Fig. 1).

\section{Individuals at high risks of symptomatic COVID-19 infections}

Population-based studies demonstrated that the incidence of COVID-19 cases in those aged 20-59 years was similar to that among older adults [6,59] (Additional file 1: Table S4). Our meta-analysis showed the cumulative incidence was 139-161 per 100,000 persons among those aged 20-59 years, which was comparable to incidence in those aged $\geq 60$ years ( 195 per 100,000 persons) (Additional file 1: Fig. S2). These working-age adults had a higher risk of acquiring COVID-19 symptomatic infection possibly because of their large number of contacts at work and in the community [60]. Additionally, they contribute to maintenance of societal functions and economic well-being; and they generally provide care for children. Given these considerations, individuals aged 20-59 years without underlying conditions $(n=576.4$ million) were included in Tier 5 based on both utilitarian and egalitarian principles (Fig. 1).

Population-based sero-epidemiological studies also reported lower seroprevalence in children than in adults [61, 62]. Whether this reflects lower susceptibility of children to infection in general, or similar infection rates, but much higher proportions with asymptomatic disease, or rather the effect of school closures, the implemented strict social distancing measures, or a self-protective behavior of the population remains unclear. Modeling studies found conflicting results about the effect of interventions targeted at children on SARS-CoV-2 transmission at the community level [63, 64], suggesting that there is still uncertainty surrounding fundamental epidemiological features of COVID-19 (e.g., children's infectiousness $[65,66]$, susceptibility to infection $[64,67]$, and probability of developing symptoms) [68]. To ensure the continuity of educational activities, and reduce transmission, school-age children ( $n=190.2$ million) are recommended for vaccination in Tier 6 based on both utilitarian and egalitarian principles (Fig. 1).

The incidence of COVID-19 was lower in younger children. However, the severity among young children has not been fully addressed. Verdoni et al. reported an outbreak of a novel severe Kawasaki-like disease in children related to COVID-19 in Italy, which raised concerns about the impact of the pandemic on younger children [69]. Considering such possible post-infectious inflammatory syndrome as Kawasaki-like disease, younger children aged $\leq 5$ years $(n=98.7$ million), which are priority groups for influenza vaccination, are recommended in Tier 6 as well, based on egalitarian principles of prioritizing the most vulnerable individuals (Fig. 1).

\section{Estimated size of target population of the phased universal vaccination program}

To maintain essential societal functions, the target population of vaccination was estimated at 49.7 million (Tiers 1 and 2, Fig. 1 and Fig. 2). An additional 563.6 million persons were included in the target population if the goal of vaccination was extended to reduce the number 


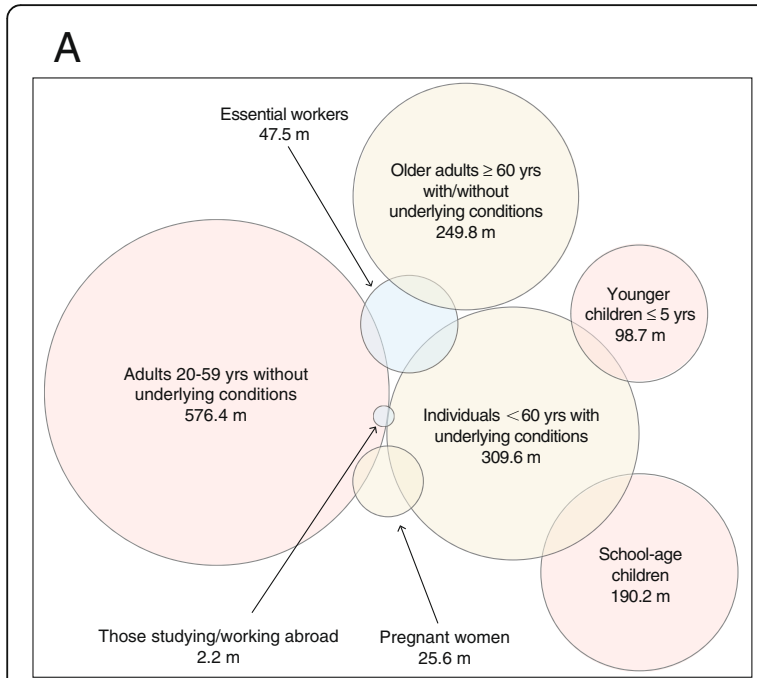

Goals of a COVID-19 vaccination program
B

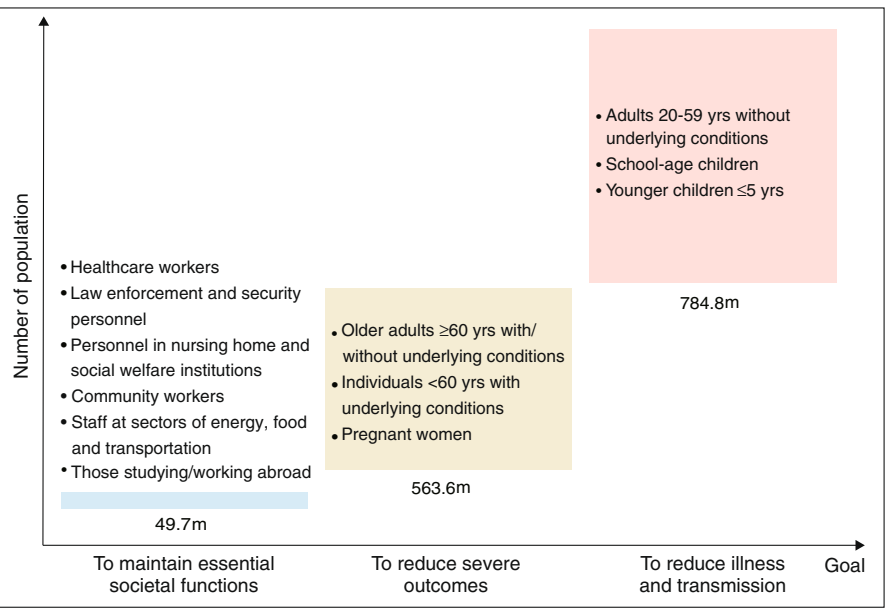

Primary goal

Secondary goal

Tertiary goal

Fig. 2 Estimated size of target population for the COVID-19 vaccination program by goal. a Overlap of target population groups. b Estimated number of targeted individuals excluding the overlaps between groups. Note that $\mathrm{m}$ denotes million

of severe COVID-19 cases (Tiers 3 and 4, Fig. 1 and Fig. 2). Along with the increase of vaccine supply, the remaining 784.8 million persons could be further targeted for vaccination to reduce the total number of COVID-19 symptomatic cases and potentially halt transmission (Tiers 5 and 6, Fig. 1 and Fig. 2). In terms of vaccination tiers (from Tier 1 to Tier 6), a total of 10.7, 39.0, 162.9, 400.6, 524.1, and 260.7 million persons were included in the target population (Figs. 1, 3 and 4).

Given 10 million doses administered per day, and a two-dose vaccination schedule, it will likely take about 7 months to vaccinate $70 \%$ of the overall population. However, only 1 week would be required to vaccinate individuals working in critical infrastructure sectors (Tier 1 and 2), three weeks for Tier 3, two months for Tier 4, about 2 months for Tier 5, and 1 month for Tier 6 (Fig. 4, and Additional file 1: Fig. S1). With an expected 0.61 billion doses produced this year [10], and given a fixed $70 \%$ uptake rate among tiers, the estimated vaccine supply could cover individuals in Tiers $1-3$ and half of individuals in Tier 4 given a two-dose vaccination schedule.

Sensitivity analyses show it will take 8 months to vaccinate $90 \%$ of individuals given 10 million doses administered each day; 1.8 years to vaccinate $70 \%$ of individuals given 3 million doses administered each day; 2.3 years to vaccinate 90\% of individuals given 3 million doses administered each day (Additional file 1: Figs. S3-S5). It will take around 4 months to vaccinate $90 \%$ and $70 \%$ of individuals respectively, if the capacity of COVID-19 vaccination delivery was scaled up to 20 million doses administered each day (Additional file 1: Figs. S6-S7).

\section{Discussion}

In the absence of specific antiviral treatment for COVID-19, vaccination likely represents the most promising way to control the COVID-19 pandemic. However, even if a COVID-19 vaccine becomes available, initial supplies will inevitably be limited. Supply issues could persist in the long term, due to huge global demand and limited production capacity. Almost everyone can potentially benefit from vaccination because of residual high susceptibility to SARS-CoV-2 infection. Considering different goals of a future vaccination program, changes in vaccine supplies, various levels of responsibility of population groups to the COVID-19 pandemic responses and essential services, as well as the risk of severe outcome and illness, we recommend a phased universal COVID-19 vaccination program for mainland China. Workers in critical sectors, including healthcare workers, law enforcement and security personnel, personnel in nursing home, and social welfare institutes, as well as sectors of energy, water, food, and transportation, and overseas workers/students (49.7 million) are the main candidates to receive high priority for vaccination, in order to maintain essential societal functions. Subsequently, we propose to extend the vaccination program to older adults, pregnant women, and those with underlying medical conditions (563.6 million), in order to reduce severe outcomes of COVID-19. Finally, working-age adults, school-age children, and younger children (784.8 million) could be vaccinated in order to reduce symptomatic COVID-19 infections, and/or to stop SARS-CoV-2 transmission. 


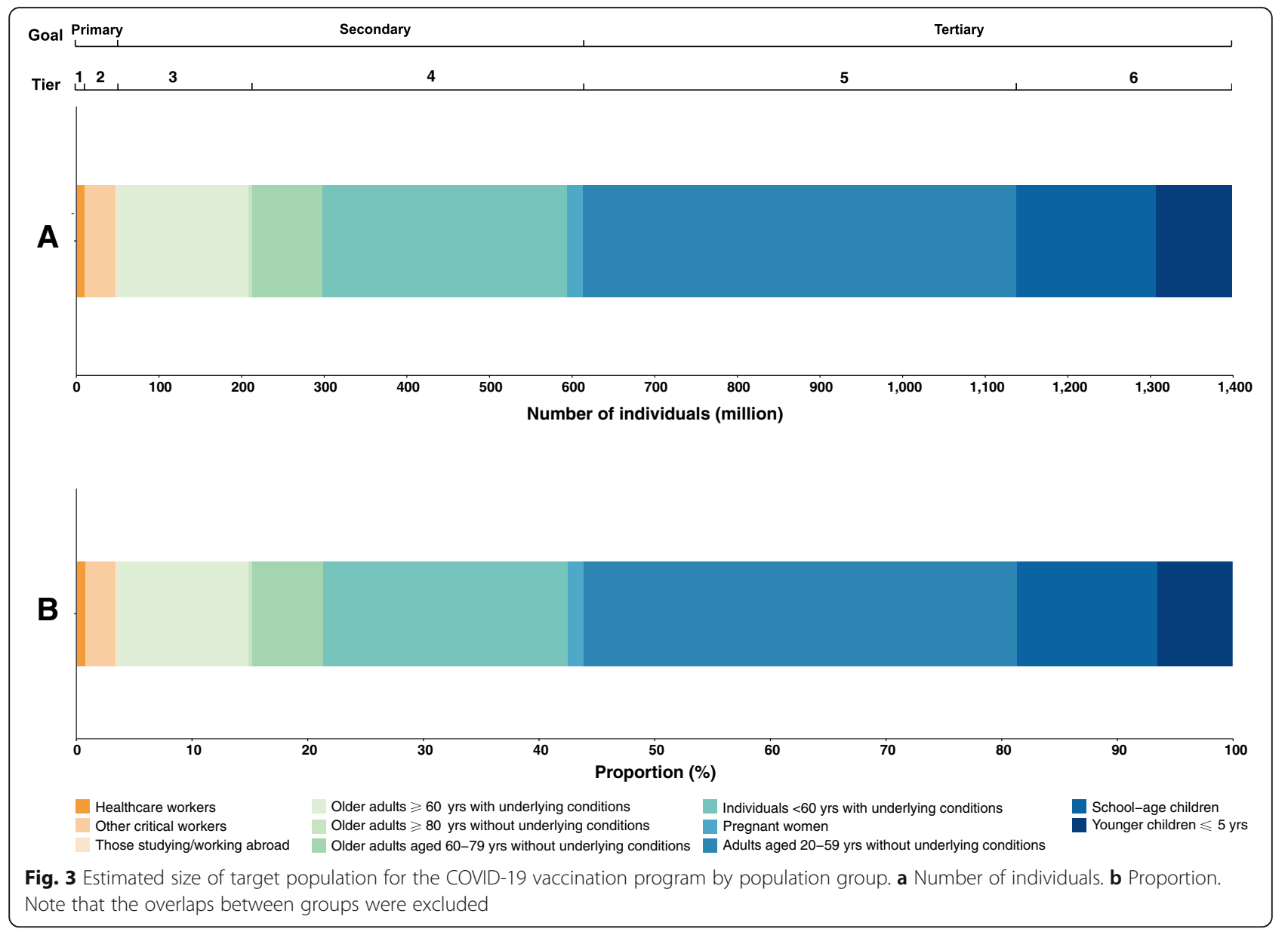

Target population groups are further grouped into vaccination tiers from 1 to 6 , with Tier 1 having the highest priority. Even though individuals within a tier have equal priority for vaccination, it may be necessary to sub-prioritize vaccination of groups within a tier if initial vaccine supplies are severely limited. For instance, cold-chain workers who have been particularly affected by COVID-19 and are often linked to workplace transmission could thus be vaccinated before other personnel within Tier 2 [70]. Other examples are represented by individuals aged $\geq 80$ years or older with underlying conditions, who may be vaccinated before other personnel within Tier 3 or by individuals of $<60$ years of age with $\geq 2$ underlying conditions who may represent a subprioritized category within their Tier [20]. Further studies are warranted to examine the sub-prioritization within each vaccination tier. Although other factors like smoking, being male, and being an ethnic minority were found to be risk factors of severe outcome and deaths from COVID-19 in previous studies [71-73], they were not accounted for when determining priority population here due to consideration of equity and feasibility of vaccination.
The Joint Committee on Vaccination and Immunisation (JCVI) in the UK largely prioritizes individuals for vaccination based on age, considering simple age-based programs to be easier to implement and thus have a higher chance of achieving a high vaccine uptake [74]. As of December 1, 2020, JCVI does not provide precise advice on the prioritization for frontline healthcare and social workers. On the other hand, the Framework for Equitable Allocation of COVID-19 Vaccine of the National Academy of Sciences, Engineering, and Medicine suggests that in the US priority should be given to frontline healthcare workers, and those having significant risk of severe illness or death from COVID-19 (as individuals with two or more underlying health conditions) [20]. Compared to the UK and the US, the epidemiological situation in China is quite different, with an almost entirely susceptible population to SARS-CoV-2 infection and very limited local transmission. In this context, the frontline workers and individuals studying/working abroad represent the categories at higher risk of infections in mainland China. Our advice on priority populations for a COVID-19 vaccination came under the umbrella of the WHO SAGE Values Framework for The 


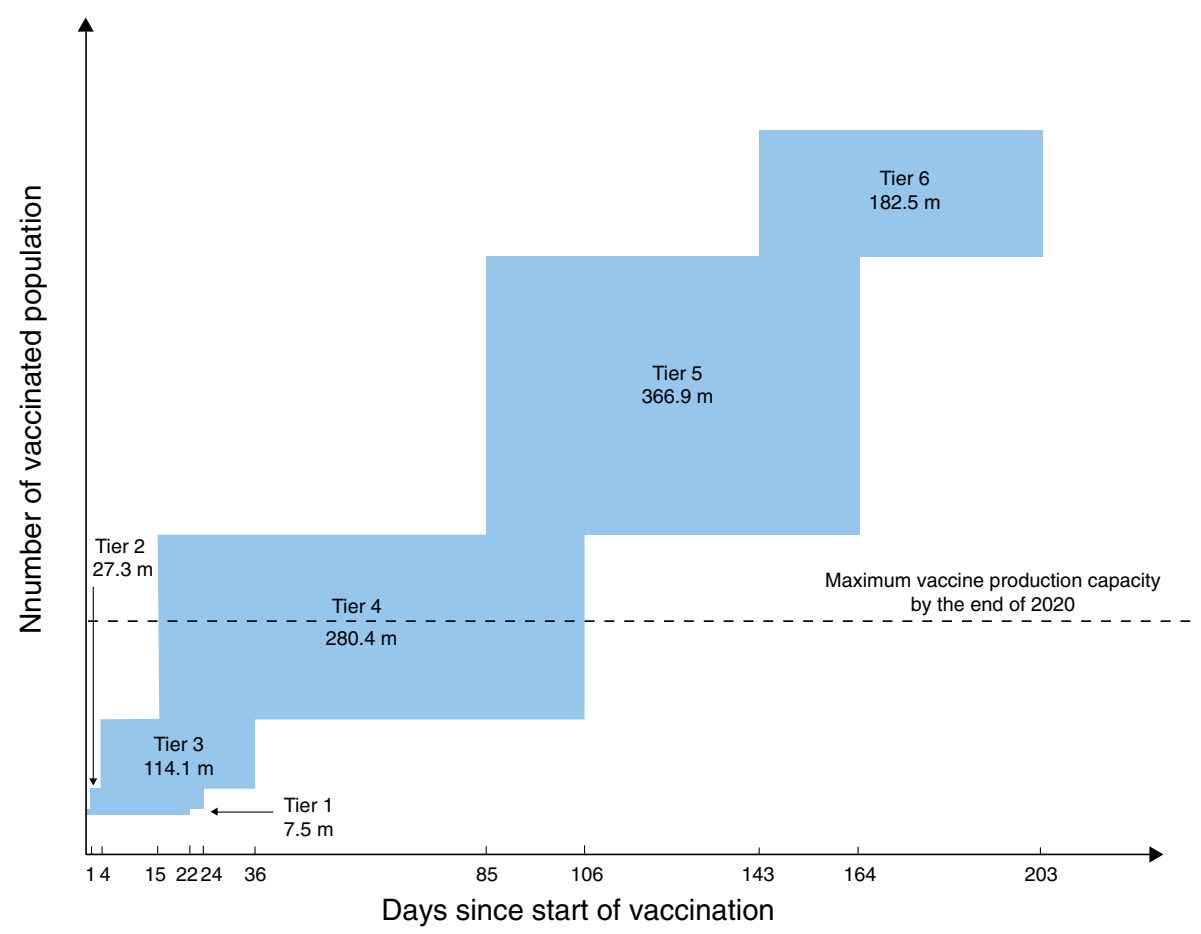

Fig. 4 Days needed to vaccinate 70\% of the target population, stratified by vaccination tier, under the assumption that 10 million doses are administered per day. Note that values reported within the square (e.g., $182.5 \mathrm{~m}$ ) denote $70 \%$ of the population size in each tier; $\mathrm{m}$ denotes million

Allocation and Prioritization of COVID-19 Vaccination [5], and took into consideration the local context and the possible goals of a COVID-19 vaccination program in China.

The majority of the current COVID-19 vaccine candidates are being trialed as two-dose schedules [9]. A total of 70 million, 789 million, and 1099 million doses are separately needed to cover $70 \%$ of individuals in critical infrastructure sectors, persons at high risk of severe outcomes of COVID-19, and persons at high risk of acquiring symptomatic illness/infections. Between 2007 and 2015 , the volume of all vaccines supplied $(n=55)$ licensed in mainland China varied from 666 million doses to 1.19 billion doses per year [75]. Several manufacturers state that a total of 0.61 billion doses of COVID-19 vaccine could be produced this year and 2.1 billion doses in 2021 [10]. Even if these candidate vaccines could be licensed and manufactured smoothly, it will take 7 months to vaccinate $70 \%$ of the general population. This is assuming an optimistic vaccine delivery rate that is over twofold higher than the maximum rate at which H1N1pdm vaccines were delivered in 2009 (3 million doses administered each day). Such a large-scale vaccination program like COVID-19 could also represent a major challenge for current the National Immunization Program in China, which is currently focused on childhood vaccination rather than on adult vaccination. The limited production capacity will likely further delay
COVID-19 vaccination programs. This dilemma is likely not unique to China, and other countries across the world, particularly in low- and middle-income regions, will face a similar challenge.

Although according to survey results [13-15], 72.591.3\% of the Chinese population aged 18 years or above appear to be willing to accept a COVID-19 vaccine, specific groups like pregnant women may be less willing to get COVID-19 vaccine due to safety concerns. These factors may delay or reduce the effective vaccine coverage. The acceptance of COVID-19 vaccination in specific segments of the population merit further studies.

Identifying individuals with underlying conditions is critical for a risk-based vaccination campaign. In China, the National Basic Public Health Service Program provides all residents with electronic health records, which have information on underlying conditions and can be queried by community healthcare centers [76]. Electronic health records as well as other medical records may be used to identify high-risk individuals with underlying conditions.

Our study has a number of limitations. First, we have qualitatively discussed the segments of the population to be prioritized in a COVID-19 vaccination program as well as the rationale behind prioritization choices. However, we could not quantitatively examine whether prioritizing older adults to reduce severe outcomes is a better choice than prioritizing working-age adults or 
school-age children to reduce illness/transmission. Mathematical modeling is urgently needed to assess both the health and economic impacts of potential vaccination strategies, and the potential to reduce for herd immunity benefits. Second, we did not consider eligibility for vaccination due to lack of efficacy and/or safety concerns that may affect specific groups such as older adults, people with pre-existing medical conditions, pregnant women, and very young children, since no vaccine has been licensed yet. Third, we did not consider real-time reactive outbreak immunization strategies because it is impossible to estimate the corresponding target population size. However, we strongly recommend use of COVID-19 vaccination during local outbreaks coupled with other non-pharmaceutical interventions in order to prevent subsequent waves of disease. Moreover, we did not discuss prioritization based on geography; the risk of COVID-19 exposure may be low in regions that have seen widespread COVID-19 activity by the time the vaccine is available and have a high level of population immunity. This may not be particularly relevant for China where the epidemic has been well controlled, but it may affect vaccine prioritization in other regions.

Because of the high burden and limited capacity for vaccine production, we have highlighted that more attention should be paid to low- and middle-income countries. The WHO SAGE Values Framework for The Allocation and Prioritization of COVID-19 Vaccination offers guidance for allocating and targeting COVID-19 pandemic vaccines, by providing six core principles and twelve objectives that further specify the six principles [5]. We tailored it to China-specific contexts accounting for the risk of illness and transmission, lessons learned from the response to the COVID-19 outbreak in Wuhan, the objectives of COVID-19 pandemic responses, and experience gained from the 2009 H1N1 pandemic vaccination program in China, in addition to the risk of severe outcomes, symptomatic illness, and transmission. Our recommendations for mainland China could be used as a template for usage of such guidelines. When a vaccine becomes available, our recommendations need to be reassessed to consider the eligibility of population subgroups based on the licensure label. They also need to be further reassessed periodically to account for changes in vaccine supply, demand, and local epidemiology. Although we propose a general framework to define vaccination priorities, the proposed vaccination program needs to be tailored locally, accounting for country-specific contexts such the objectives of the pandemic responses, the local level of transmission, the make-up of first responders, and essential workers as well as the capacity of immunization services.

\section{Conclusions}

Vaccine deployment is likely to become vitally important for the global response to the COVID-19 pandemic. Here we provide a general framework to define priority groups for a phased introduction of a universal COVID19 vaccination program. We applied this framework to mainland China and further estimated the corresponding target population sizes. The proposed vaccination program could assist Chinese policy-makers in the roll-out of a large-scale immunization program and be used as a reference for other countries, especially in low- and middle-income regions.

\section{Supplementary Information}

The online version contains supplementary material available at https://doi. org/10.1186/s12916-021-01923-8.

Additional file 1: Table S1. COVID-19 vaccine priority groups. Figure S1. Schematic diagram of vaccinating $70 \%$ of target populations by Tiers. Table S2. Characteristics of systematic reviews for persons at high risk of severe outcome of COVID-19. Table S3. Age distribution of COVID-19 cases. Table S4. Data used to estimate the COVID-19 incidence rate. Figure S2. Pooled incidence of COVID-19 cases, stratified by age. Figure S3. Sensitivity analyses on days needed to vaccinate $90 \%$ of the target population, under the assumption that 10 million doses are administered per day. Figure S4. Sensitivity analyses on days needed to vaccinate $70 \%$ of the target population, under the assumption that 3 million doses are administered per day. Figure S5. Sensitivity analyses on days needed to vaccinate $90 \%$ of the target population, under the assumption that 3 million doses are administered per day. Figure S6. Sensitivity analyses on days needed to vaccinate $70 \%$ of the target population, under the assumption that 20 million doses are administered per day. Figure S7. Sensitivity analyses on days needed to vaccinate $90 \%$ of the target population, under the assumption that 20 million doses are administered per day.

\section{Abbreviations}

COVID-19: Coronavirus disease 2019; WHO: World Health Organization; SAGE: Strategic Advisory Group of Experts on Immunization; SARS-CoV2: Novel severe acute respiratory syndrome coronavirus 2; US: United States; UK: United Kingdom; GBD: Global Burden of Diseases, Risk Factors, and Injuries Study; UN: United Nations; VE: Vaccine efficacy; $R_{0}$ : Basic reproduction number; H1N1pdm: Pandemic influenza H1N1; JCVI: The Joint Committee on Vaccination and Immunisation

\section{Acknowledgments}

We thank Dr. Yang Liu from Centre for Mathematical Modelling of Infectious Diseases, London School of Hygiene and Tropical Medicine, London, United Kingdom, for her comments to revise this article.

\section{Authors' contributions}

H.Y. conceived, designed, and supervised the study. J.Y., W.Z., H.S., X.Y., K.D., Q.Y., G.Z., H.G. and Z.C. participated in data collection. J.Y., W.Z., H.S., X.Y., K.D., and Q.Y. analyzed the data, and prepared the tables and figures. J.Y. prepared the first draft of the manuscript. H.Y., M.J., C.V., and M.A. commented on the data and its interpretation and revised the content critically. All authors contributed to review and revision and approved the final manuscript as submitted and agree to be accountable for all aspects of the work. The authors read and approved the final manuscript.

\section{Funding}

The study was supported by grants from the National Science Fund for Distinguished Young Scholars (No. 81525023), National Science and Technology Major Project of China (No. 2018ZX10201001-010, No. 2018ZX10713001-007, No. 2017ZX10103009-005), and the National Institute for Health Research (NIHR) (grant no. 16/137/109) using UK aid from the UK Government to support global 
health research. The views expressed in this publication are those of the author(s) and not necessarily those of the NIHR or the UK Department of Health and Social Care.

\section{Availability of data and materials}

All data and data source were provided in details on GitHub at https:// github.com/wenzidebanxia/target_population.

\section{Ethics approval and consent to participate}

All these data were in the public domain. All these are aggregated data, only presenting the numbers of cases or proportions/percentages, and thus are robustly anonymized data. Ethical review for the re-use of these secondary data is not required.

\section{Consent for publication}

Not applicable.

\section{Competing interests}

H.Y. has received research funding from Sanofi Pasteur, GlaxoSmithKline, Yichang HEC Changjiang Pharmaceutical Company, and Shanghai Roche Pharmaceutical Company. M.A. has received research funding from Seqirus. None of those research funding is related to COVID-19. All other authors report no competing interests.

\section{Author details}

${ }^{1}$ School of Public Health, Fudan University, Key Laboratory of Public Health Safety, Ministry of Education, Shanghai, China. ${ }^{2}$ Centre for Mathematical Modelling of Infectious Diseases, London School of Hygiene and Tropical Medicine, London, UK. ${ }^{3}$ Department of Infectious Disease Epidemiology, London School of Hygiene \& Tropical Medicine, London, UK. ${ }^{4} \mathrm{WHO}$ Collaborating Centre for Infectious Disease Epidemiology and Control, School of Public Health, Li Ka Shing Faculty of Medicine, The University of Hong Kong, Special Administrative Region, Hong Kong, China. ${ }^{5}$ Division of International Epidemiology and Population Studies, Fogarty International Center, National Institutes of Health, Bethesda, MD, USA. ${ }^{6}$ Department of Epidemiology and Biostatistics, Indiana University School of Public Health Bloomington, IN, USA. ${ }^{7}$ Laboratory for the Modeling of Biological and Socio-technical Systems, Northeastern University, Boston, MA, USA. ${ }^{8}$ Shanghai Institute of Infectious Disease and Biosecurity, Fudan University, Shanghai, China. ${ }^{9}$ Department of infectious diseases, Huashan Hospital, Fudan University, Shanghai, China.

Received: 23 September 2020 Accepted: 20 January 2021 Published online: 10 February 2021

\section{References}

1. Li Z, Chen Q, Feng L, Rodewald L, Xia Y, Yu H, Zhang R, An Z, Yin W, Chen $W$, et al. Active case finding with case management: the key to tackling the COVID-19 pandemic. Lancet. 2020;396(10243):63-70.

2. London School of Hygiene \& Tropical Medicine. COVD-19 vaccine tracker. 2020. https:/vac-Ishtm.shinyapps.io/ncov vaccine landscape/. Accessed 30 Nov 2020

3. World Health Organization. ACT-Accelerator update. 2020. https://www.who.int/ news-room/detail/26-06-2020-act-accelerator-update. Accessed 15 July 2020.

4. Johns Hopkins University. Coronavirus (COVID-19) Information and Updates. 2020. https://www.coronavirustraining.org/live-map. Accessed 15 July 2020.

5. World Health Organization. WHO SAGE Values Framework for The Allocation and Prioritization of COVID-19 Vaccination. 2020. https://apps.who.int/iris/ bitstream/handle/10665/334299/WHO-2019-nCoV-SAGE FrameworkAllocation_and_prioritization-2020.1-eng.pdf?ua=1. Accessed 22 Sept 2020

6. Pan A, Liu L, Wang C, Guo H, Hao X, Wang Q, Huang J, He N, Yu H, Lin X, et al. Association of Public Health Interventions with the epidemiology of the COVID-19 outbreak in Wuhan, China. JAMA. 2020;323(19):1915-23.

7. Beijing Municipal Health Commission. Weekly Epidemic Report (from June 8, 2020 to June 14, 2020). 2020. http://wjw.beijing.gov.cn/English/ HealthServices/WeeklyEpidemicReport/202007/t20200703_1938589.html. Accessed 15 July 2020

8. Ministry of Science and Technology of the People's Republic of China. Response to COVID-19 pandemic coordinated call for BRICS multilateral projects 2020. 2020. http://www.most.gov.cn/tztg/202007/t20200717_15 7932.htm. Accessed 2 Aug 2020.
9. World Health Organization. Draft landscape of COVID-19 candidate vaccines 2020. https://www.who.int/publications/m/item/draft-landscape-of-covid-19candidate-vaccines. Accessed 30 Nov 2020.

10. The Central People's Government of the People's Republic of China. COVID19 vaccine development, priority populations and pricing ... the lastest release! 2020. http://www.gov.cn/fuwu/2020-10/20/content_5552857.htm. Accessed 30 Nov 2020.

11. SOHU. At least 60,000 people in China have tried the COVID-19 vaccine. Can we wait for it this winter? https://m.sohu.com/a/432706359_115571/ ?pvid=000115_3w_a. Accessed 30 Nov 2020.

12. NetEase Finance. Sinopharm has submitted an application for the marketing of COVID-19 vaccine. 56,000 people have been vaccinated and left China without any infection. https://money.163.com/20/1126/07/FSBG26030025 9DLP.html. Accessed 30 Nov 2020.

13. Fu C, Wei Z, Pei S, Li S, Sun X, Liu P. Acceptance and preference for COVID19 vaccination in health-care workers (HCWs). medRxiv. 2020; https://doi.org/ 10.1101/2020.04.09.20060103.

14. Wang J, Jing $R$, Lai $X$, Zhang $H$, Lyu Y, Knoll MD, Fang $H$. Acceptance of COVID-19 vaccination during the COVID-19 pandemic in China. Vaccines. 2020;8(3):482

15. Lazarus JV, Ratzan SC, Palayew A, Gostin LO, Larson HJ, Rabin K, Kimball S, El-Mohandes A. A global survey of potential acceptance of a COVID-19 vaccine. Nat Med. 2020:1-4.

16. Reed C, Biggerstaff M, Finelli L, Koonin LM, Beauvais D, Uzicanin A, Plummer A, Bresee J, Redd SC, Jernigan DB. Novel framework for assessing epidemiologic effects of influenza epidemics and pandemics. Emerg Infect Dis. 2013;19(1):85-91.

17. Freitas ARR, Napimoga M, Donalisio MR. Assessing the severity of COVID-19. Epidemiol Serv Saude. 2020;29(2):e2020119.

18. Centers for Disease Control and Prevention. Interim updated planning guidance on allocating and targeting pandemic influenza vaccine during an influenza pandemic. 2018. https://www.cdc.gov/flu/pandemic-resources/ pdf/2018-Influenza-Guidance.pdf. Accessed 24 Jul 2020.

19. Johns Hopkins Center for Health Security. Interim Framework for COVID-19 Vaccine Allocation and Distribution in the United States. 2020. https://www. centerforhealthsecurity.org/our-work/pubs_archive/pubs-pdfs/2020/200819vaccine-allocation.pdf. Accessed 10 Aug 2020.

20. National Academies of Sciences $E$, and Medicine. Framework for Equitable Allocation of COVID-19 Vaccine. 2020. https://doi.org/10.17226/25917 Accessed 30 Nov 2020.

21. The Central People's Government of the People's Republic of China. Guidance on 2009 influenza pandemic vaccination programme in China. 2009. http:// www.gov.cn/zwgk/2009-09/23/content_1424257.htm. Accessed 26 July 2020.

22. Zimmerman RK. Rationing of influenza vaccine during a pandemic: ethical analyses. Vaccine. 2007;25(11):2019-26.

23. Chatterjee $P$, Anand $T$, Singh $K$, Rasaily $R$, Singh R, Das $S$, Singh $H$, Praharaj I, Gangakhedkar RR, Bhargava B, et al. Healthcare workers \& SARS-CoV-2 infection in India: A case-control investigation in the time of COVID-19. Indian J Med Res. 151(5):459-67.

24. Kimball A, Hatfield KM, Arons M, James A, Taylor J, Spicer K, Bardossy AC, Oakley LP, Tanwar S, Chisty Z, et al. Asymptomatic and Presymptomatic SARS-CoV-2 infections in residents of a long-term care skilled nursing facility - King County, Washington, march 2020. MMWR Morb Mortal Wkly Rep. 2020;69(13):377-81.

25. The State Council Information Office of the People's Republic of China. White paper on "China's National Defense in the New Era". 2019. http://www.scio. gov.cn/zfbps/32832/Document/1660314/1660314.htm. Accessed 15 July 2020.

26. National Bureau of Statistics. Tabulation the 2010 Population Census of the People's Republic of China. http://www.stats.gov.cn/tjsj/pcsj/rkpc/6rp/ indexch.htm. Accessed 30 Nov 2020.

27. Ministry of National Defense of the People's Republic of China. White Paper on "China's National Defense In 2006". 2011. http://www.mod.gov.cn/ regulatory/2011-01/06/content_4617808_4.htm. Accessed 30 Nov 2020.

28. Li Q. Research on the optimal allocation of police human resources based on big data. People's Public Security University of China; 2020.

29. Ministry of Commerce. PRC. Concise statistics on China's foreign labor service cooperation business from January to October 2020. http://hzs. mofcom.gov.cn/article/date/202011/20201103018497.shtml. Accessed 30 Nov 2020.

30. Ministry of Education of the People's Republic of China. The considerations of college entrance examination delay; How to arrange the resumption of 
classes? http://www.moe.gov.cn/jyb_xwfb/s5147/202004/t20200401_437149. html. Accessed 30 Nov 2020.

31. Clark A, Jit M, Warren-Gash C, Guthrie B, Wang HHX, Mercer SW, Sanderson C, McKee M, Troeger C, Ong KL, et al. Global, regional, and national estimates of the population at increased risk of severe COVID-19 due to underlying health conditions in 2020: a modelling study. Lancet Glob Health. 2020;8(8):e1003-e17.

32. United Nations. World population prospects 2019. https://population.un.org/ wpp/Download/Standard/Population/. Accessed 15 Jul 2020.

33. National Health Commission of the People's Republic of China. China Health Statistical Yearbook: Peking Union Medical College Press; 2020.

34. Wang YP, lang J, Zhu J, Zhou GX, Miao L, L. D. Analysis of the perinatal death and related male/female ratio of hospital delivery in China during 1988-1992. J Pract Obstet Gynecol. 2001;17:173-174.

35. Liu B, Gao ES. Risk factors for spontaneous abortion of Chinese married women at reproductive age. China Public Health. 2002;18(7):890-2.

36. Ministry of Education of China. Statistics Yearbook of Education in 2019 2020. http://www.moe.gov.cn/s78/A03/moe_560/jytjsj_2019/qg/. Accessed 12 Aug 2020.

37. Cohen J. 'Absolutely remarkable': No one who got Moderna's vaccine in trial developed severe COVID-19. https://www.sciencemag.org/news/2020/11/ absolutely-remarkable-no-one-who-got-modernas-vaccine-trial-developedsevere-covid-19. Accessed 30 Nov 2020

38. Li Q, Guan X, Wu P, Wang X, Zhou L, Tong Y, Ren R, Leung KSM, Lau EHY, Wong JY, et al. Early transmission dynamics in Wuhan, China, of novel coronavirus-infected pneumonia. N Engl J Med. 2020;382(13):1199-207.

39. Fine P, Eames K, Heymann DL. "herd immunity": a rough guide. Clin Infect Dis. 2011;52(7):911-6.

40. Hogan A, Winskill P, Watson O, Walker P, Whittaker C, Baguelin M, Haw D, Lochen A, Gaythorpe K, Ainslie K et al. Modelling the allocation and impact of a COVID-19 vaccine. https://spiral.imperial.ac.uk:8443/handle/10044/1/82 822. Accessed 30 Nov 2020.

41. The Central People's Government of the People's Republic of China. Report of H1N1 pandemic influenza vaccination from Ministry of Health. 2010. http://www.gov.cn/gzdt/2010-04/13/content_1579310.htm. Accessed 15 July 2020.

42. Seale $H$, Kaur R, Wang Q. Acceptance of a vaccine against pandemic influenza A ( $\mathrm{H} 1 \mathrm{~N} 1)$ virus amongst healthcare workers in Beijing, China. Vaccine. 2011;29:1605-10.

43. Wagner AL, Zhang Y, Mukherjee B, Ding Y, Wells EV, Boulton ML. The impact of supplementary immunization activities on the epidemiology of measles in Tianjin, China. Int J Infect Dis. 2016;45:103-8.

44. Pranata R, Lim MA, Yonas E, Vania R, Lukito AA, Siswanto BB, Meyer M. Body mass index and outcome in patients with COVID-19: a dose-response metaanalysis. Diab Metab. 2020;S1262-S3636(20):30097-5.

45. Zhou Y, Yang Q, Chi J, Dong B, Lv W, Shen L, Wang Y. Comorbidities and the risk of severe or fatal outcomes associated with coronavirus disease 2019: a systematic review and meta-analysis. Int J Infect Dis. 2020;99:47-56.

46. Zheng Z, Peng F, Xu B, Zhao J, Liu H, Peng J, Li Q, Jiang C, Zhou Y, Liu S, et al. Risk factors of critical \& mortal COVID-19 cases: a systematic literature review and meta-analysis. J Inf Secur. 2020;81:e16-25.

47. Fang X, Li S, Yu H, Wang P, Zhang Y, Chen Z, Li Y, Cheng L, Li W, Jia H, et al. Epidemiological, comorbidity factors with severity and prognosis of COVID-19: a systematic review and meta-analysis. Aging. 2020;12(13):12493-503.

48. Zhang J, Wang X, Jia X, Li J, Hu K, Chen G, Wei J, Gong Z, Zhou C, Yu H, et al. Risk factors for disease severity, unimprovement, and mortality in COVID-19 patients in Wuhan, China. Clin Microbiol Infec. 2020;26(6):767-72.

49. Yang J, Zheng Y, Gou X, Pu K, Chen Z, Guo Q, Ji R, Wang H, Wang Y, Zhou $Y$. Prevalence of comorbidities and its effects in patients infected with SARSCoV-2: a systematic review and meta-analysis. Int J Infect Dis. 2020;94:91-5.

50. Singh AK, Gillies CL, Singh R, Singh A, Chudasama Y, Coles B, Seidu S, Zaccardi F, Davies MJ, Khunti K. Prevalence of co-morbidities and their association with mortality in patients with COVID-19: a systematic review and meta-analysis. Diabetes Obes Metab. 2020;22(10):1915-24.

51. Kovalic AJ, Satapathy SK, Thuluvath PJ. Prevalence of chronic liver disease in patients with COVID-19 and their clinical outcomes: a systematic review and meta-analysis. Hepatol Int. 2020;14(5):612-20.

52. Pastick KA, Nicol MR, Smyth E, Zash R, Boulware DR, Rajasingham R, McDonald EG. A systematic review of treatment and outcomes of pregnant women with COVID-19 - a call for clinical trials. Open Forum Infec Dis. 2020;7(9):ofaa350.
53. Panahi L, Amiri M, Pouy S. Risks of novel coronavirus disease (COVID-19) in pregnancy; a narrative review. Arch Acad Emerg Med. 2020;8(1):e34.

54. Centers for Disease Control and Prevention. People who are at higher risk for severe illness. 2020. https://www.cdc.gov/coronavirus/2019-ncov/needextra-precautions/people-with-medical-conditions.html?CDC_AA_refVal= https\%3A\%2F\%2Fwww.cdc.gov\%2Fcoronavirus\%2F2019-ncov\%2Fneedextra-precautions\%2Fgroups-at-higher-risk.html. Accessed July 152020.

55. Liu M, Gao Y, Zhang Y, Shi S, Chen Y, Tian J. The association between severe or dead COVID-19 and autoimmune diseases: a systematic review and meta-analysis. J Inf Secur. 2020;81:e93-e5.

56. Gao Y, Chen Y, Liu M, Shi S, Tian J. Impacts of immunosuppression and immunodeficiency on COVID-19: a systematic review and meta-analysis. J Inf Secur. 2020;81(2):e93-e5.

57. Wu JT, Leung K, Bushman M, Kishore N, Niehus R, de Salazar PM, Cowling BJ, Lipsitch M, Leung GM. Estimating clinical severity of COVID-19 from the transmission dynamics in Wuhan, China. China Nat Med. 2020;26:506-10.

58. Banerjee A, Pasea L, Harris S, Gonzalez-Izquierdo A, Torralbo A, Shallcross L, Noursadeghi M, Pillay D, Sebire N, Holmes C, et al. Estimating excess 1-year mortality associated with the COVID-19 pandemic according to underlying conditions and age: a population-based cohort study. Lancet. 2020; 395(10238):1715-25.

59. Stokes EK, Zambrano LD, Anderson KN, Marder EP, Raz KM, El Burai FS, Tie Y, Fullerton KE. Coronavirus disease 2019 case surveillance - United States, January 22-may 30, 2020. MMWR Morb Mortal Wkly Rep. 2020;69(24):759-65.

60. Zhang J, Klepac P, Read JM, Rosello A, Wang X, Lai S, Li M, Song Y, Wei Q, Jiang $\mathrm{H}$, et al. Patterns of human social contact and contact with animals in Shanghai, China. Sci Rep. 2019;9(1):15141.

61. Pollán M, Pérez-Gómez B, Pastor-Barriuso R, Oteo J, Hernán MA, PérezOlmeda M, Sanmartín JL, Fernández-García A, Cruz I, Fernández de Larrea N, et al. Prevalence of SARS-CoV-2 in Spain (ENE-COVID): a nationwide, population-based seroepidemiological study. Lancet. 2020; 396(10250):535-44.

62. Stringhini S, Wisniak A, Piumatti G, Azman AS, Lauer SA, Baysson H, De Ridder D, Petrovic D, Schrempft S, Marcus K, et al. Seroprevalence of antiSARS-CoV-2 IgG antibodies in Geneva, Switzerland (SEROCoV-POP): a population-based study. Lancet. 2020:396(10247):313-9.

63. Davies NG, Klepac P, Liu Y, Prem K, Jit M, Group CC-W, Eggo RM. Agedependent effects in the transmission and control of COVID-19 epidemics. Nat Med. 2020;26(8):1205-11.

64. Zhang J, Litvinova M, Liang Y, Wang Y, Wang W, Zhao S, Wu Q, Merler S, Viboud C, Vespignani A, et al. Changes in contact patterns shape the dynamics of the COVID-19 outbreak in China. Science. 2020;368(6498):1481-6.

65. Hu S, Wang W, Wang Y, Litvinova M, Luo K, Ren L, Sun Q, Chen X, Zeng G, Li J et al. Infectivity, susceptibility, and risk factors associated with SARS-CoV2 transmission under intensive contact tracing in Hunan, China. medRxiv. 2020;https://doi.org/10.1101/2020.07.23.20160317.

66. Szablewski CM, Chang KT, Brown MM, Chu VT, Yousaf AR, Anyalechi N, Aryee PA, Kirking HL, Lumsden M, Mayweather E, et al. SARS-CoV-2 transmission and infection among attendees of an overnight camp Georgia, June 2020. MMWR Morb Mortal Wkly Rep. 2020;69(31):1023-5.

67. Viner RM, Mytton OT, Bonell C, Melendez-Torres GJ, Ward J, Hudson L, Waddington C, Thomas J, Russell S, Klis Fvd et al. Susceptibility to SARSCoV-2 infection amongst children and adolescents compared with adults: a systematic review and meta-analysis. medRxiv. 2020;https://doi.org/10.1101/ 2020.05.20.20108126.

68. Poletti P, Tirani M, Cereda D, Trentini F. Probability of symptoms and critical disease after SARS-CoV-2 infection. arXiv. 2020;https:/arxiv.org/abs/2006.08471.

69. Verdoni L, Mazza A, Gervasoni A, Martelli L, Ruggeri M, Ciuffreda M, Bonanomi E, D'Antiga L. An outbreak of severe Kawasaki-like disease at the Italian epicentre of the SARS-CoV-2 epidemic: an observational cohort study. Lancet. 2020;395(10239):1771-8.

70. Liu P, Yang M, Zhao X, Guo Y, Wang L, Zhang J, Lei W, Han W, Jiang F, Liu WJ, et al. Cold-chain transportation in the frozen food industry may have caused a recurrence of COVID-19 cases in destination: successful isolation of SARS-CoV-2 virus from the imported frozen cod package surface. Biosaf Health. 2020;2(4):199-201.

71. Williamson EJ, Walker AJ, Bhaskaran K, Bacon S, Bates C, Morton CE, Curtis HJ, Mehrkar A, Evans D, Inglesby P, et al. Factors associated with COVID-19related death using OpenSAFELY. Nature. 2020;584(7821):430-6.

72. Khunti K, Singh AK, Pareek M, Hanif W. Is ethnicity linked to incidence or outcomes of covid-19? BMJ. 2020;369:m1548. 
73. The Institute for Fiscal Studies. Are some ethnic groups more vulnerable to COVID-19 than others? 2020. https://web.archive.org/web/20200502130148/ https://www.ifs.org.uk/inequality/chapter/are-some-ethnic-groups-morevulnerable-to-covid-19-than-others/. Accessed 15 July 2020.

74. The Joint Committee on Vaccination and Immunisation. JCVI: updated interim advice on priority groups for COVID-19 vaccination. 2020. https:// www.gov.uk/government/publications/priority-groups-for-coronaviruscovid-19-vaccination-advice-from-the-jcvi-25-september-2020/jcvi-updatedinterim-advice-on-priority-groups-for-covid-19-vaccination. Accessed $30 \mathrm{Nov}$ 2020.

75. Zheng Y, Rodewald L, Yang J, Qin Y, Pang M, Feng L, Yu H. The landscape of vaccines in China: history, classification, supply, and price. BMC Infect Dis. 2018;18(1):502.

76. National Basic Public Health Service Program Management Platform. Standard of resident health records management service. http://www. nbphsp.org.cn/jbgw/jkda/. Accessed 30 Nov 2020.

\section{Publisher's Note}

Springer Nature remains neutral with regard to jurisdictional claims in published maps and institutional affiliations.

Ready to submit your research? Choose BMC and benefit from:

- fast, convenient online submission

- thorough peer review by experienced researchers in your field

- rapid publication on acceptance

- support for research data, including large and complex data types

- gold Open Access which fosters wider collaboration and increased citations

- maximum visibility for your research: over $100 \mathrm{M}$ website views per year

At $\mathrm{BMC}$, research is always in progress.

Learn more biomedcentral.com/submissions 